\title{
CMS Tracker Operational Experience
}

\section{Francesco Fiori ${ }^{* \dagger}$}

National Taiwan University (NTU), Taipei (TW)

E-mail: francesco.fioriecern.ch

The CMS Tracker was repaired, recalibrated and commissioned successfully for the second run of Large Hadron Collider. In 2015 the Tracker performed well with improved hit efficiency and spatial resolution compared to Run I and operated smoothly at lower temperatures after the successful recommissioning of the cooling system. This year the detector is expected to withstand luminosities that are beyond its design limits and will need a combined effort of both online and offline team to yield the high quality data that is required to reach our physics goals. We present the experience gained during the second run of the LHC and show the latest performance results of the CMS Tracker.

The 25th International workshop on vertex detectors

September 26-30, 2016

La Biodola, Isola d'Elba, ITALY

*Speaker.

${ }^{\dagger}$ on behalf of the CMS Collaboration 


\section{Introduction}

In this paper is discussed the operational experience with the CMS Tracker gained during the long period of data taking at the Large Hadron Collider (LHC), with a particular focus on the Run II.

This data taking period started in early 2015 and is characterized by a substantial improvement of the accelerator performance with respect to the Run I period. The beam energy has been raised to $6.5 \mathrm{TeV}$ to provide pp collisions at a center of mass energy of $13 \mathrm{TeV}$ in order to enhance the discovery potential of LHC experiments. Also the instantaneous luminosity has been greatly improved, reaching values above $1.5 \times 10^{34} \mathrm{~cm}^{-2} \mathrm{~s}^{-1}$, about $30 \%$ above the original design value. The bunch spacing has been reduced from 50 to $25 \mathrm{~ns}$, with a maximum number of 2232 colliding bunches in the accelerator; in these conditions up to 40 primary interactions per bunch crossing are produced. Most of the developments have been deployed in 2016, permitting to collect over 37 $\mathrm{fb}^{-1}$ of proton-proton collisions in a single year, already exceeding the total collected in Run I and in 2015 (Figure 1), the plan is to achieve $100 \mathrm{fb}^{-1}$ by the end of 2018 .

This outstanding improvement of the beam conditions made 2016 a very challenging year for the

CMS Integrated Luminosity, pp

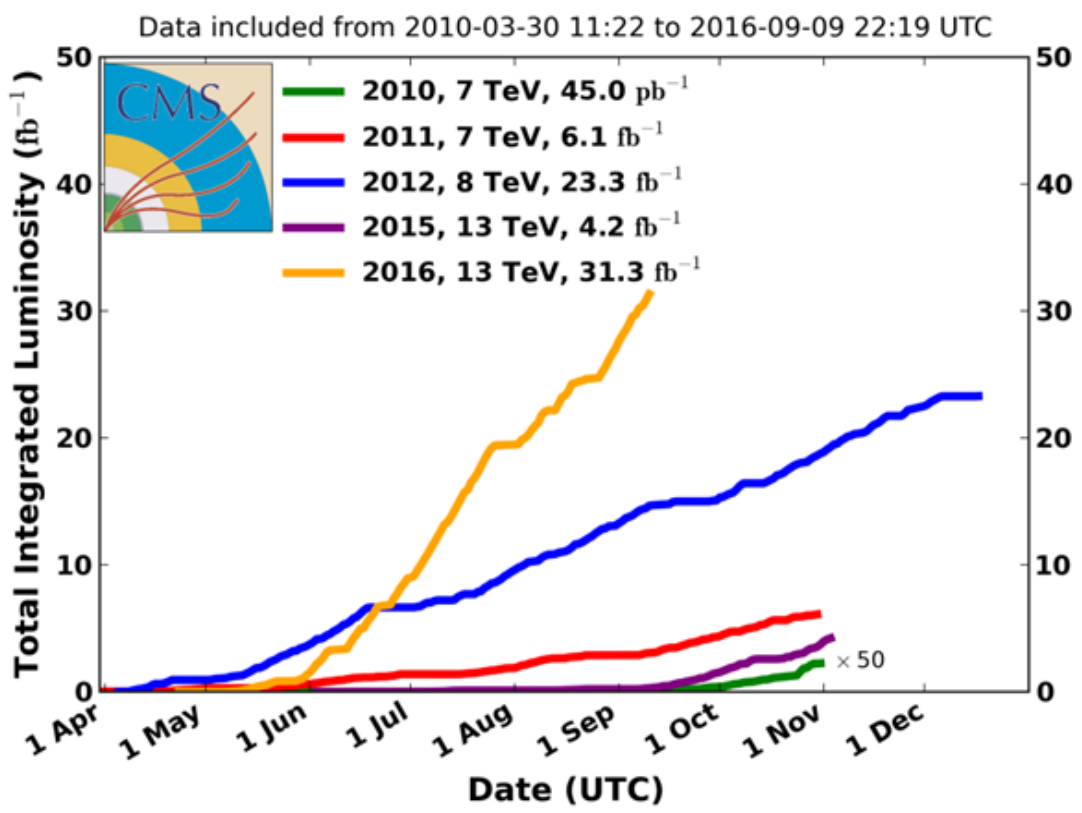

Figure 1: Evolution of integrated luminosity in Run I and Run II data taking periods.

CMS detector that faced a significant increase of occupancy and data rate, of particular impact on the Tracker system, being the closest to the interaction point.

\section{The CMS Tracker}

The CMS Tracker is entirely based on silicon sensors, it is composed of a pixel detector, which is closest to the interaction point, and a micro-strip detector. The total coverage in pseudo-rapidity 
extends up to \pm 2.5 (Figure 2).

The Pixel detector, fundamental for primary and secondary vertex reconstruction, is made of three

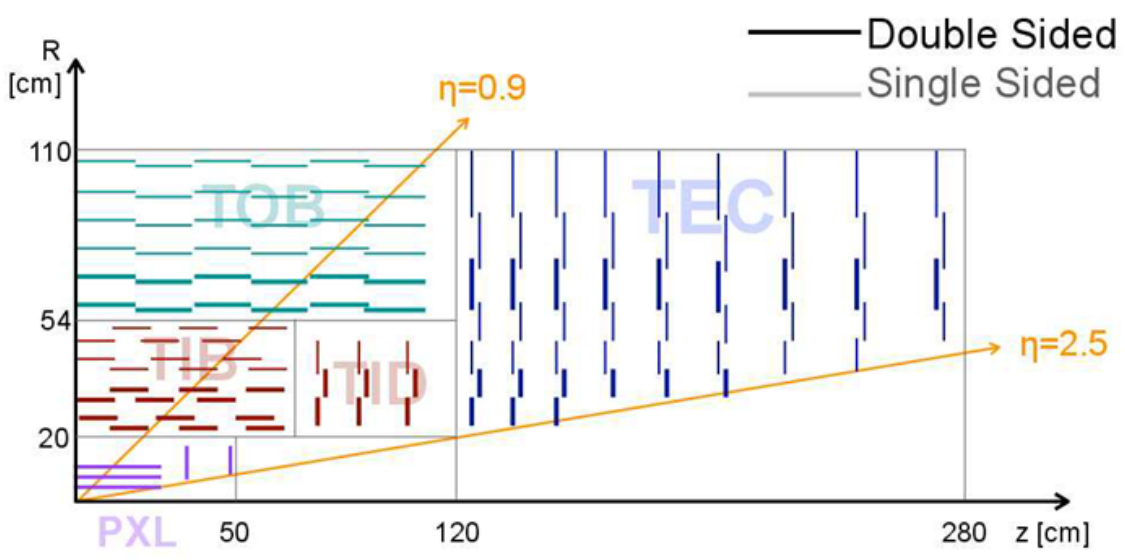

Figure 2: Schematic view of the CMS Tracker in the r-z plane, the picture shows $1 / 4$ of the CMS Tracker. The acronyms refer to different Tracker subdetectors: Pixel (PXL), Tracker Inner Barrel (TIB), Tracker Outer Barrel (TOB), Tracker Inner Disks (TID) and Tracker End-Cap (TEC).

barrel layers and two encap disks on each side. The first layer is placed at $4.3 \mathrm{~cm}$ from the beam line. The barrel is equipped with 768 modules (Figure 3), each composed of 16 analog read-out chips (ROCs) bump-bonded on $\mathrm{n}^{+}$-on-n sensors, each ROC serving 52 x 80 pixels. The end-cap disks are composed of 24 blades arranged in a turbine-like geometry, each made of two panels placed back to back and containing a total number of 45 ROCs (Figure 3). The total number of pixels is 66 millions, assuring a three hits coverage in the full Tracker acceptance.

The strip detector, made of about $200 \mathrm{~m}^{2}$ of active area, is the largest particle detector based on silicon sensors ever built. It is composed of an inner detector, made of four barrel layers and three disks in each end, and an outer part made of 6 barrel layer and 9 end-cap disks on each side. Sensors have 300 or $500 \mu \mathrm{m}$ thickness (the thinner ones are used in the inner part), made on $\mathrm{p}^{+}$-on-n silicon with a strip pitch from 83 to $205 \mu \mathrm{m}$. The read-out is analog and the total number of electronic channels is $9.6 \mathrm{M}$.

A detailed description of the CMS Tracker can be found in [1].

\section{Activities during the long shutdown}

In the period between Run I and Run II periods, known as Long Shutdown 1 (LS 1), several activities have been pursued to guarantee that data taking can be resumed in the best possible condition. The Pixel detector has been extracted and repaired on the surface. Several faulty connections to power cables and optical converters have been repaired and a set of 59 modules, damaged during Run I data taking, have been completely replaced with spare components. The Pixel system has been then reinserted in CMS in late 2014.

A lot of work has been done on the cooling system; new cooling plant cabinets and heat exchangers have been installed. A new dry gas injection system has been introduced. Also the insulation has been greatly improved, permitting to operate safely the Tracker at low temperature: $-15^{\circ} \mathrm{C}$ for the 


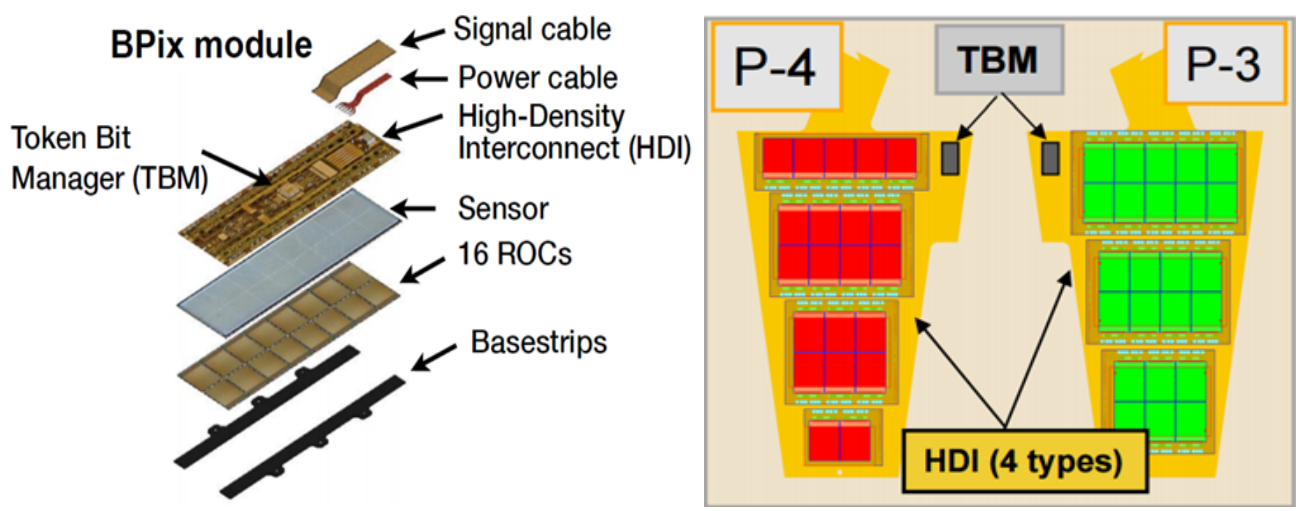

Figure 3: Left: schematic view of the barrel pixel module components. Right:ROC arrangement in the forward pixel panels.

Strip detector and $-10^{\circ} \mathrm{C}$ for the Pixel system. The Run I operational temperatures were $4^{\circ} \mathrm{C}$ and $0^{\circ} \mathrm{C}$ respectively.

\section{Pixel detector status and performance in Run II}

In general the overall status of the Pixel detector is nicely improved with respect to Run I. The replacement of bad modules cured almost completely the forward disks, the fraction of working pixels in Run II is above $99.95 \%$, with only two faulty ROCs in total. The situation for the barrel part is not so positive. After repair during LS 1 and reinstallation, 16 modules arranged in a sector of Layer 2 could not be powered at the design B-field of 3.8 T. Nevertheless, the overall fraction of working pixels in Run II is $98.2 \%$, exceeding the Run I value of $97.7 \%$.

The bias voltage in Run II has been increased from $150 \mathrm{~V}$ to $200 \mathrm{~V}$ to compensate for radiation damage effects.

The hit finding efficiency measured in Run II is well above 99\% for outer layers and disks (Figure 4). For Layer 1, that is closer to the interaction point, a decreasing trend is observed as a function of instantaneous luminosity. This effect is expected given the actual design of the analog chip, suffering from dynamic inefficiency at the highest data rate; the current lowest measurement of efficiency in Layer 1 is $95.4 \%$ at an instantaneous luminosity of $1.2 \times 10^{34} \mathrm{~cm}^{-2} \mathrm{~s}^{-1}$.

For what concerns the hit resolution, no degradation of performance has been observed with respect to Run I; the average value is less then $10 \mu$ min the $r-\phi$ plane. Two methods are used for this measurement: a generic method based on a fast estimate of the hit position, and a template method, based on a detailed simulation of cluster shapes for different track incidence angles, the latter giving the best measurement. The values obtained with the two methods are 9.5 and $8.5 \mu \mathrm{m}$, respectively, very stable as a function of integrated luminosity (Figure 4). A fundamental ingredient to achieve the best possible hit resolution is the measurement of the Lorentz angle and the monitoring of its evolution with integrated luminosity. Two different methods are used for the measurement in the barrel and in the forward disks: the grazing angle method and the minimum cluster size method, respectively. The first method is based on the selection of tracks with a very shallow angle with respect to the module surface, to measure the drift length as a function of the depth in the sensor. The second method is based on the fact that the minimum of the cluster size in the forward regions 

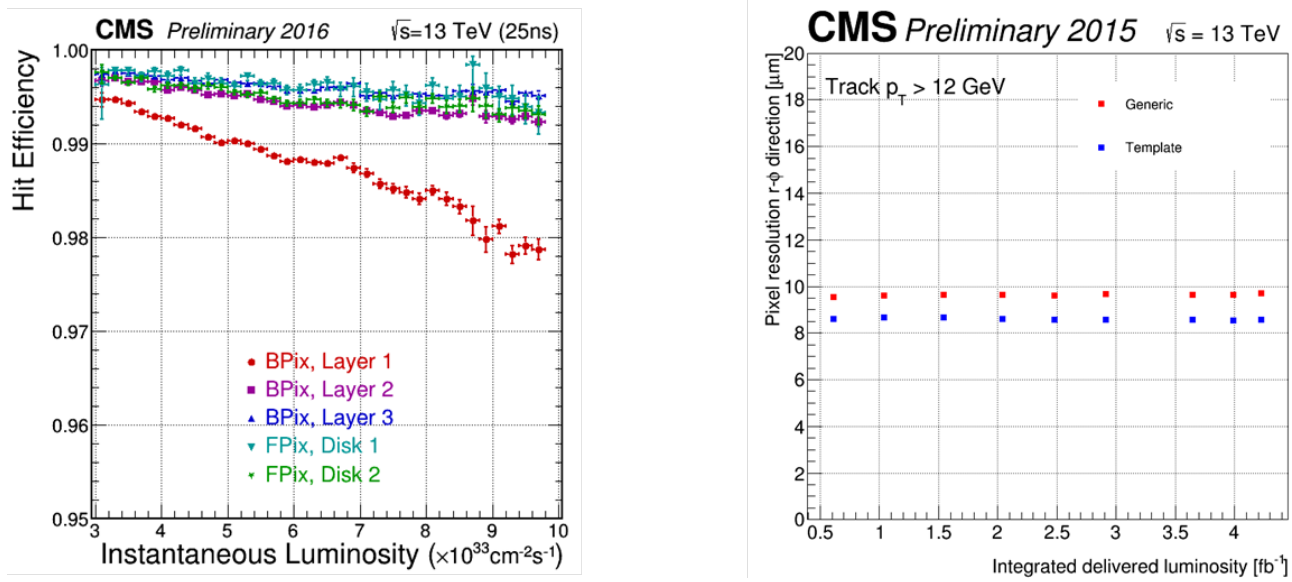

Figure 4: Left: Hit finding efficiency. Right: hit position resolution.

is obtained with tracks parallel to the drift direction. Results for the grazing angle method are presented in Figure 5.

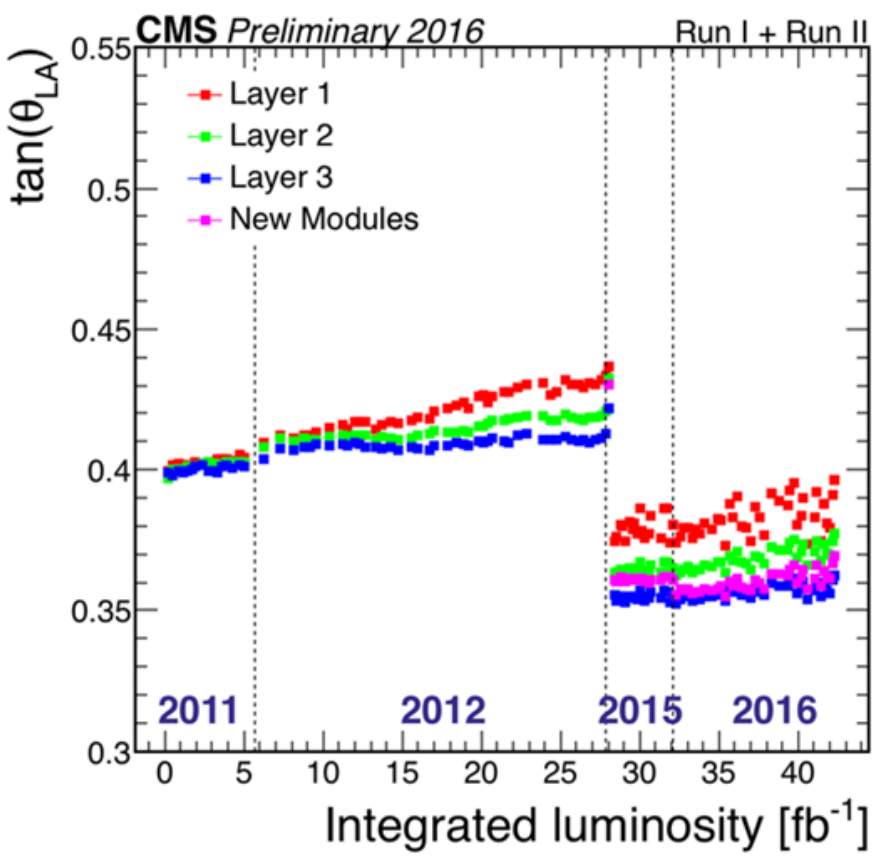

Figure 5: Lorentz angle measurement in Run I and Run II data. The step observed at the starting of 2015 is due to the increase of bias voltage, from $150 \mathrm{~V}$ to $200 \mathrm{~V}$ at the beginning of 2015 . The new modules referred in the figure are those replaced during the Long Shoutdown.

\section{Strip detector status and performance in Run II}

The number of bad components in the Strip detector is monitored on a run by run basis and with different granularity, faulty modules, read-out chips or even single strips are identified and 
recorded during data taking. The overall fraction of bad components recorded in Run II, in terms of single strips, is in the range 3.6-3.8\%, showing a very good stability with respect to the Run I period. However, in late 2015 and half of 2016 data taking, the Strip detector suffered a major issue affecting hit finding efficiency showing up as an excess of low signal-over-noise ratio clusters associated with loss of tracking hits. This feature was dependent on the instantaneous luminosity and more prominent in regions with the highest occupancy.

This behaviour has been traced back to saturation in the pre-amplifier stage finally cured chang-
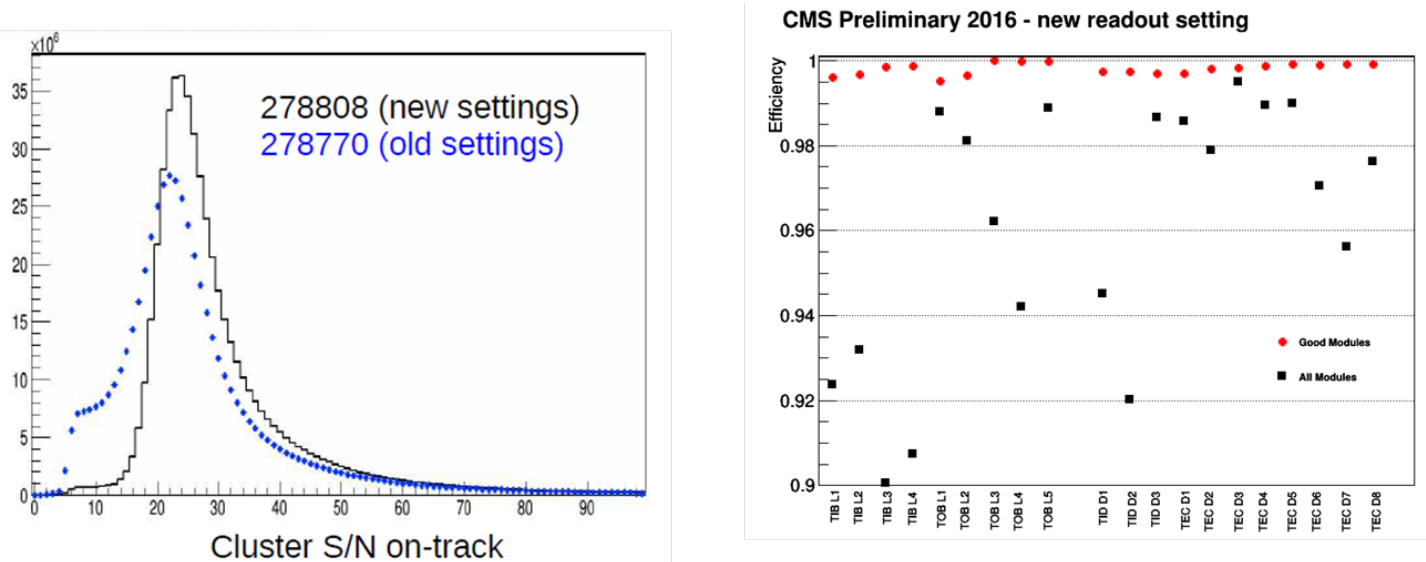

Figure 6: Left: effect of the new read-out chip setting on the signal-over-noise $(\mathrm{S} / \mathrm{N})$ ratio distribution. Right: hit efficiency after deployment of new settings; red dots represent the efficiency computed on good modules, black dots correspond to the efficiency computed including also faulty components.

ing the hardware configuration of the read-out chip. Immediately after the deployment of the new parameters the inefficiency has completely disappeared. Figure 6 shows the effect of this dynamic inefficiency and the result after deploying the new settings on the $\mathrm{S} / \mathrm{N}$ distribution for clusters used in track reconstruction. Affected data (about $22 \mathrm{fb}^{-1}$ ) will be reprocessed using an ad-hoc mitigation algorithm to reduce to the minimum the effect on data quality. However the overall impact, even before any mitigation was applied, was not dramatic.

Once the new settings had been in place, the performance of the Strip detector went back to what was seen in Run I: hit finding efficiency well above $99.5 \%$ (Figure 6) independently of the instantaneous luminosity.

\section{Alignment}

A basic ingredient to achieve the best possible performance of the Tracker is the alignment. An imperfect knowledge of the actual module positions will result in loss of hit resolution and efficiency. A module level alignment has been redone in 2016 using 1 million collision events and cosmic data. Figure 7 shows the effect of the new alignment on the track impact parameter with respect to the primary vertex.

One of the primary causes of unwanted movements of the Tracker structure are magnet cycles: as an example, the pixel detector can move by about $0.3 \mathrm{~mm}$ in the beam direction and $0.1 \mathrm{~mm}$ in the transverse plane after a magnet ramp, reducing significantly the precision of hit position 


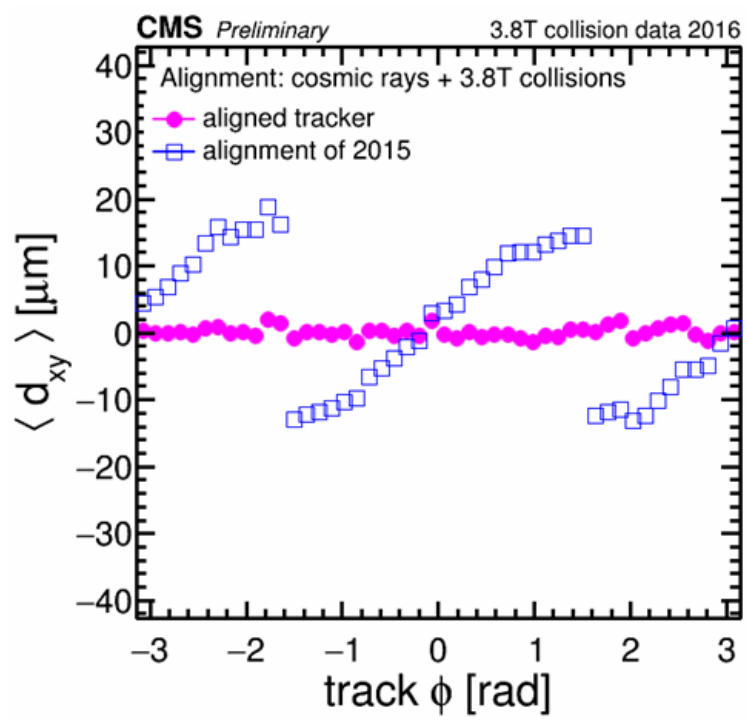

Figure 7: Transverse impact parameter of tracks with respect to the primary vertex as a function of the track momentum $\phi$ coordinate in different alignment conditions.

measurements. To compensate for this effect a new automatic alignment procedure is in place since middle 2016: the position of large structures (i.e barrel layers and end-cap disks) is monitored on a run by run basis and adjusted when necessary. Figure 8 shows the effect of automatic alignment; after a magnet cycle the residual movements in the transverse direction are of the order of $10 \mu \mathrm{m}$, reduced to a few $\mu \mathrm{m}$ after the automatic correction.

Before alignment After alignment
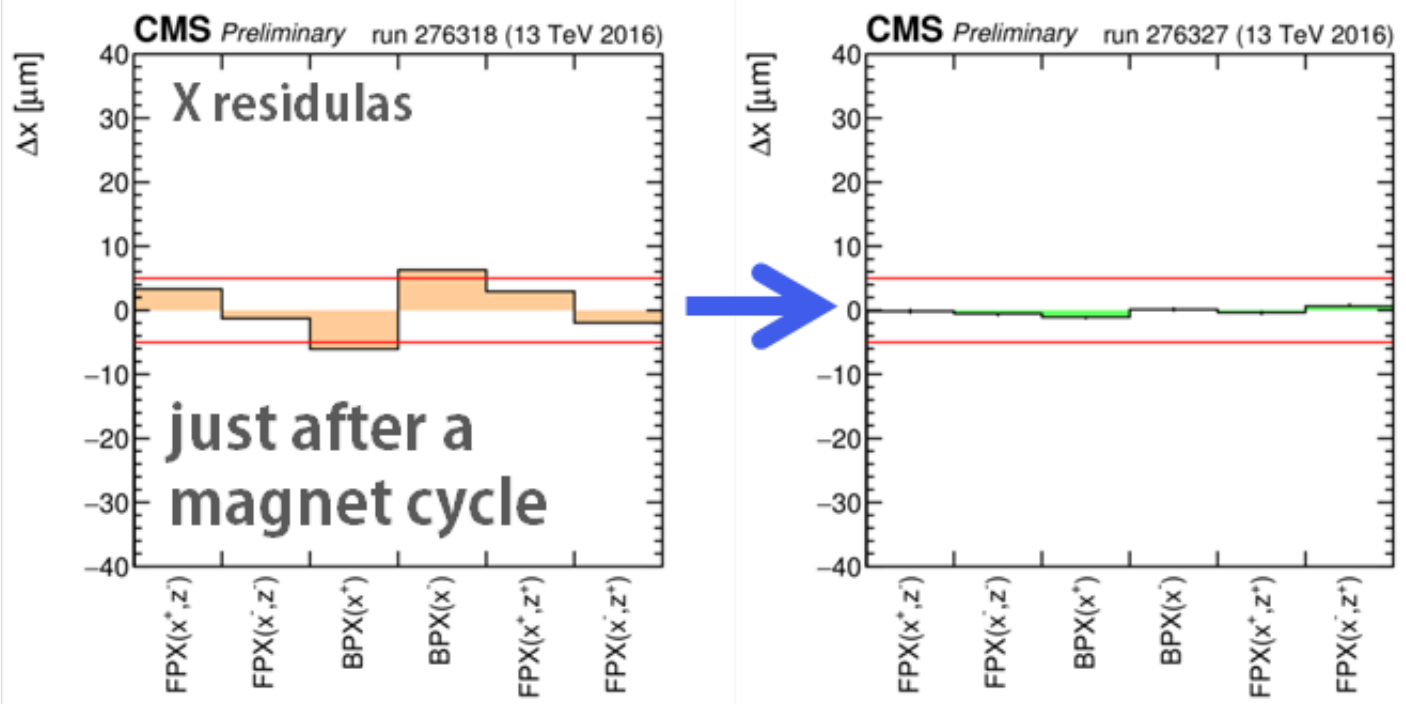

Figure 8: Automatic alignment result after a magnet cycle. In the $x$ axis labels FPX and BPX refer respectively to Forward Pixel and Barrel Pixel. 


\section{Track reconstruction performance}

The track reconstruction performance is presented in terms of the primary vertex resolution and reconstruction of invariant mass distributions of particles using only Tracker information.

The primary vertex resolution is computed with the split track method. Tracks belonging to the
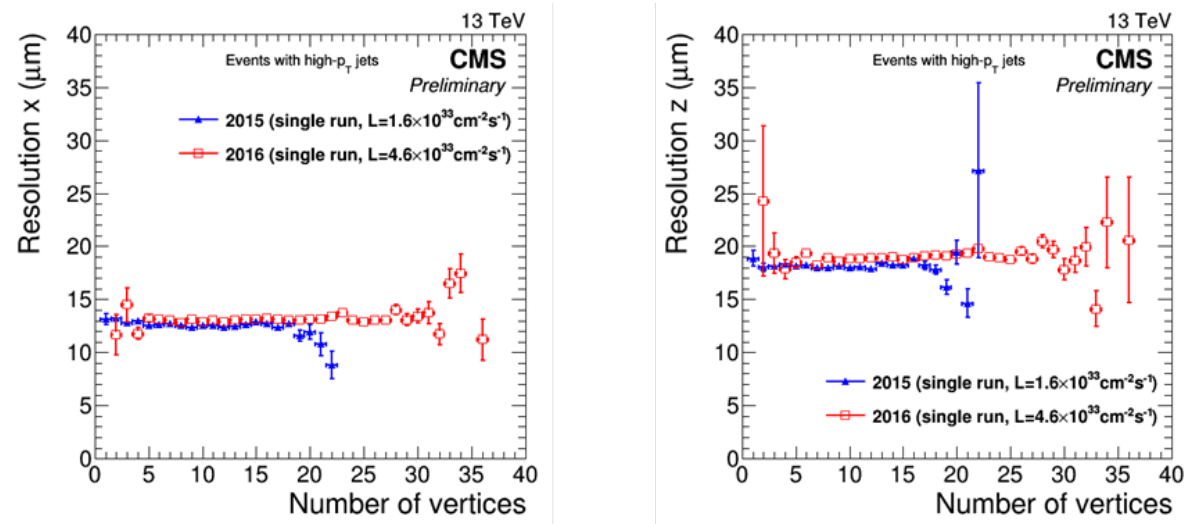

Figure 9: Primary vertex resolution in the transverse plane (left) and along the beam axis (right).

same vertex are divided randomly in two sets with a compatible transverse momentum spectrum. To extract a resolution measurement, for each set a primary vertex is reconstructed and the vertex positions are compared. Results of this procedure are shown in Figure 9 for the transverse and longitudinal resolution with respect to the beam axis. For the transverse direction the average value is about $13 \mu \mathrm{m}$, while $19 \mu \mathrm{m}$ is measured for the longitudinal direction. Both measurements are compatible with the Run I results, showing no degradation of performance.

For what concerns the invariant mass measurement and resolution, V0 channels as $K_{s} \rightarrow \pi \pi$ and $\Lambda \rightarrow p \pi$ are used (Figure 10). The central values and widths of the resonances are in very good agreement with the expected values.
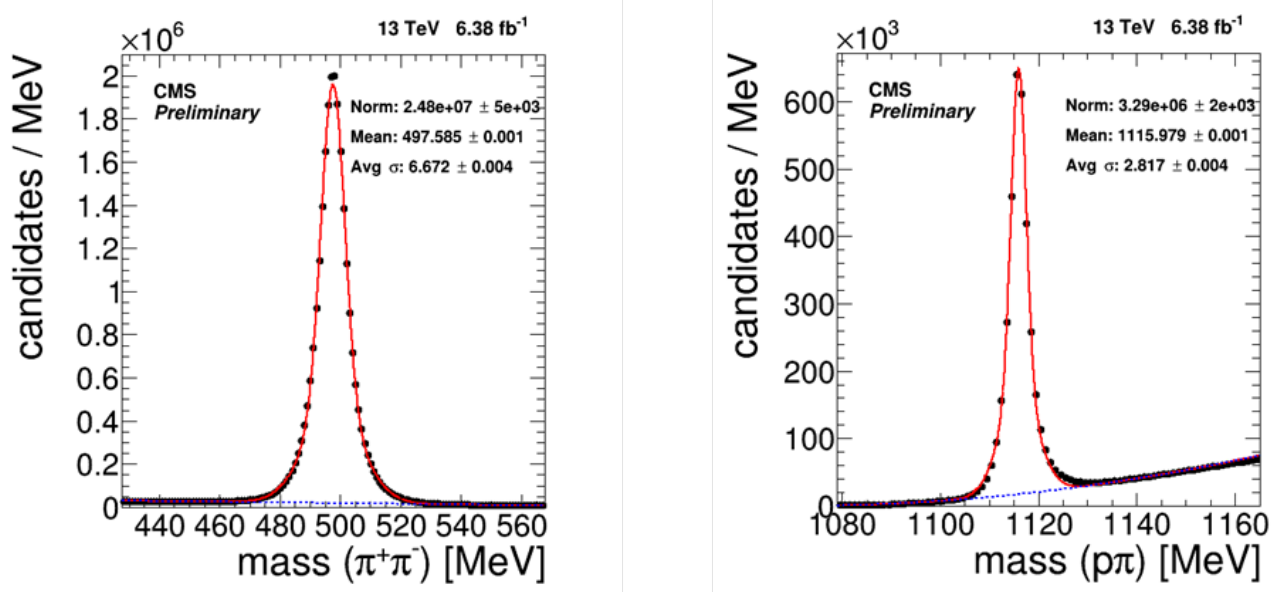

Figure 10: $K_{S} \rightarrow \pi \pi$ (left) and $\Lambda \rightarrow p \pi$ (right) invariant mass distributions. 


\section{Pilot Blade and the Phase I upgrade}

In early 2017 a new pixel detector will be installed in CMS for the, so called, phase I upgrade [2]. The new detector will have four barrel layers and three disks on each side, furthermore the read-out chip has been completely redesigned: it will be a digital chip with increased data handling performance to cure completely the dynamic inefficiency effect seen in the current data.

To prepare at best the upgrade a pilot blade (Figure 11) equipped with the new modules has been

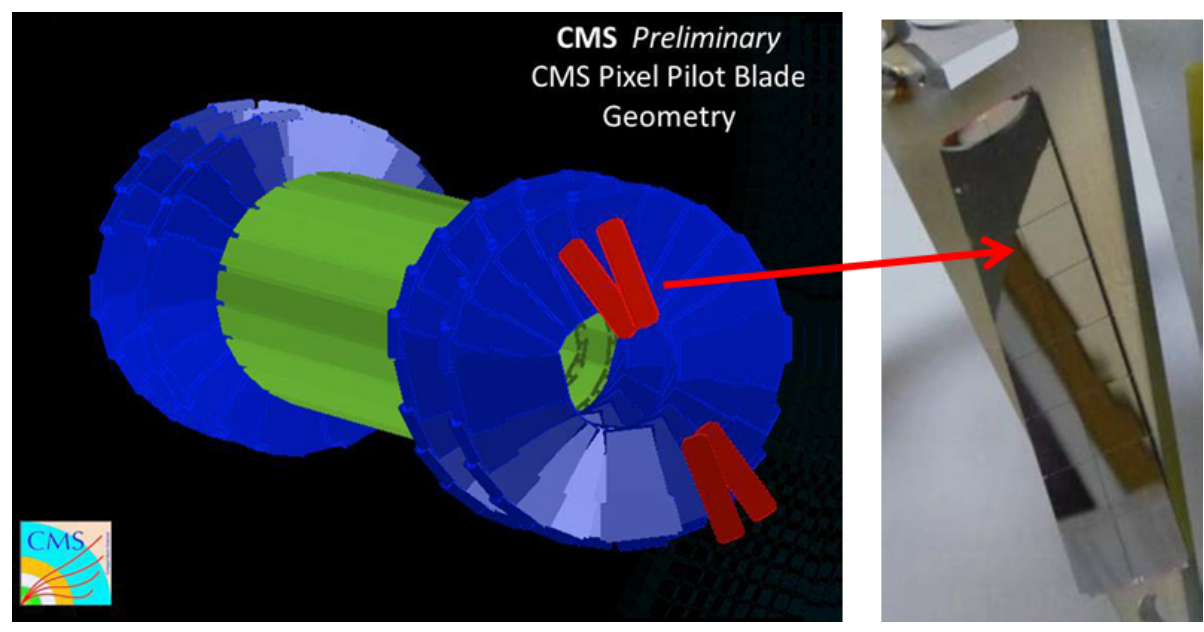

Figure 11: Pilot blade installation in the forward pixel system.

installed in the pixel forward detector in 2014 and included in the central data acquisition system in Run II. It is made of four modules installed in the forward region as shown in Figure 11. Even if not all the ROCs are commissioned and the hits in the pilot blade are not considered in track reconstruction, the pilot system gave a fundamental input in view of the commissioning of the full phase I detector foreseen for early 2017. As an example we could test the tools and procedure to achieve a good time alignment of the new detector.

\section{Summary and conclusions}

CMS is successfully taking physics data in Run II, even at the highest LHC luminosity beyond the original design. The Tracker detector is running stably with very good performance, comparable to the Run I period.The only major issue encountered in Run II data taking has been the strip dynamic inefficiency, that has been completely cured bringing back Tracking performance to Run I values.

The pilot system continues to provide valuable information in view of the Phase I upgrade of the pixel detector in 2017. In summary the CMS tracker is providing good quality data and is ready to do the same for the rest of 2016, looking forward to the new pixel detector and the highest LHC luminosities.

\section{References}

[1] The CMS Collaboration, CMS Tracker technical design report, CERN-LHCC-98-06 
[2] The CMS collaboration, CMS Technical Design Report for the Pixel Detector Upgrade, CERN-LHCC-2012-016 